\title{
Computer Simulations of Diffusional Reactions in Complex Steels
}

\author{
John ÅGREN \\ Division of Physical Metallurgy, The Royal Institute of Technology, S-100 44 Stockholm, Sweden. \\ (Received on August 26, 1991; accepted in final form on October 25, 1991)
}

\begin{abstract}
The development of a software package DICTRA for simulation of diffusional reactions in multicomponent alloys and a databank for multicomponent diffusivities is described. Applications concerning heat treatment of low-alloy steels and martensitic stainless steels are discussed.
\end{abstract}

KEY WORDS: multicomponent alloys; diffusion; thermodynamics; austenite/ferrite; carbide dissolution.

\section{Introduction}

The microstructural changes during heat treating of steel involve various diffusional processes and consequently simulation of such changes must be based on diffusion calculations. In the present report the effect of alloying and multicomponent interactions and their role in microstructural evolution will be emphasized. As input data we will need thermodynamic properties and multicomponent diffusion data. We will not try to predict the shape or morphology of particles but rather impose a simple shape and then calculate the growth rate for this hypothetic shape. We will thus be able to answer questions regarding the effect of alloying on transformation rate provided that the shape of particles is sufficiently simple.

The present author started to work along those lines a decade ago ${ }^{1)}$ and the first version of the software developed was applied to the $\alpha-\gamma$ transformation in dual-phase steels. ${ }^{2)}$ In this report the basic ideas behind the calculations will be presented as well as some of the further developments. Some recent examples of applications will be discussed.

\section{Thermodynamic Basis}

In order to calculate driving forces a thermodynamic description of the steel under consideration is needed. Such descriptions are now available for a number of binary and ternary and even some quaternary systems by means of the CALPHAD type of analyses, see for example Refs. 3)-6). It should also be mentioned that it is possible to perform calculations even in higherorder systems, which have not been assessed, simply by combining the descriptions of the lower-order systems. For cases where the lower-order systems are well characterized one usually obtains quite reasonable results by such extrapolations. Softwares like THERMO$\mathrm{CALC}^{7)}$ for calculation of thermodynamic properties and multicomponent phase diagrams enable the metallurgist to apply such data to practical problems with a moderate effort.

In the present calculations the assumption of local equilibrium at a moving phase interface will be applied. This should be a fairly reasonable approximation in many situations of practical interest. However, it should be emphasized that this assumption is readily modified in order to take into account various effects that cause departure from equilibrium. Such modifications were recently discussed by the present author. ${ }^{8)}$ Nevertheless, in the present work the THERMO-CALC software will be applied in order to calculate the local equilibrium at a moving phase interface.

In a binary isothermal system under atmospheric pressure the equilibrium tieline is uniquely determined from the temperature and may be directly calculated. In a system with $n$ components one has to specify $n-2$ conditions in addition to temperature and pressure in order to pose a problem with a unique solution and to calculate a tieline. There is thus a degeneracy in the choice of tieline and some principle is needed in order to choose among the infinite number of tielines. In an ordinary equilibrium calculation the tieline that makes the massbalance obeyed for each species is chosen. For the case of a phase transformation the tieline that obeys the flux-balance equations, see next section, is chosen. One thus has to consider $n-2$ chemical potentials as unknowns and vary them, for example by the NewtonRaphson technique, until one finds the correct tieline. In each iteration it is then necessary to calculate the equilibrium for a given set of chemical potentials.

\section{Kinetic Basis}

\subsection{Flux-balance Equations}

The flux balances at a moving phase interface between an $\alpha$ and $\beta$ phase may be written

$$
v^{\alpha} c_{k}^{\alpha}-v^{\beta} c_{k}^{\beta}=J_{k}^{\alpha}-J_{k}^{\beta} \quad k=1,2, \ldots, n
$$

where $v^{\alpha}$ and $v^{\beta}$ are the interface migration rate as measured in the local frame of reference in the $\alpha$ and $\beta$ phase respectively. $c_{k}^{\alpha}$ and $c_{k}^{\beta}$ are the concentrations (mole 
per volume) in $\alpha$ and $\beta$ close to the phase interface and $J_{k}^{\alpha}$ and $J_{k}^{\beta}$ are the corresponding diffusional fluxes. As a local frame of reference we will apply the number-fixed frame of reference with respect to the substitutional elements defined by

$$
\sum J_{k}^{\alpha}=0
$$

and a similar expression for the $\beta$ phase. The summation is performed over the substitutional elements only. It is always possible to derive a relation between $v^{\alpha}$ and $v^{\beta}$ and to eliminate one of them and one of the flux-balance equations. We thus have only $n-1$ independent fluxbalance equations from which $v^{\alpha}$ or $v^{\beta}$ and the $n-2$ unknown chemical potentials may be determined.

\subsection{Diffusion}

The diffusion in a multicomponent system may be expressed by the Fick-Onsager law

$$
J_{k}=\sum_{j=1}^{n-1} D_{k j}^{n} \nabla c_{j}
$$

The above equation holds in both $\alpha$ and $\beta . D_{k j}^{n}$ denotes the components of the diffusion-coefficient matrix in the phase under consideration when species $n$ is chosen as solvent, i.e. is excluded from the summation. $\nabla c_{j}$ is the concentration gradient of species $j$. Since $D_{k j}^{n}$ may be expected to vary strongly with composition one has to rely on numerical methods to solve the diffusion equation

$$
\frac{\partial c_{k}}{\partial t}=-\operatorname{div}\left(J_{k}\right)
$$

where div denotes the divergence operator. In the present work a method developed by Ågren was applied. ${ }^{9)}$

\section{Multicomponent Diffusion Data Bank}

As mentioned, multicomponent diffusion data are needed in order to perform the calculations. The fact that the $D_{k j}^{n}$ matrix generally is a strong function of composition presents a severe obstacle. The situation is similar to the one that the metallurgist runs into when looking for information on phase equilibria in multicomponent alloys; the experimental information is simply missing. Only a few sections of ternary phase diagrams have been determined experimentally. For higher order systems the information is almost completely missing. Not even a huge experimental work would help to remedy the situation because it is selfevident that it is impossible to cover experimentally all the possible variations in the multidimensional composition and temperature space.

One thus has to rely on extrapolations and interpolations of the sparse experimental data. As in the case of thermodynamic properties, such extrapolations should be based on reasonably simple models that mimic the physical behavior of the system rather than just power-series expansions fitted to the experimental data.

Such an approach was outlined by Andersson and Agren ${ }^{10)}$ and formed the basis for Åkermark's ${ }^{11)}$ recent analysis of the experimental data for the fcc phase in the
$\mathrm{Fe}-\mathrm{Cr}-\mathrm{Ni}$ system. We will now review his results.

Andersson and Ågren applied the simple vacancy model of diffusion, see for example the monograph by Kirkaldy and Young, ${ }^{12)}$ and for the diffusional flux of species $k$ relative the lattice-fixed frame of reference the following expression was postulated in an alloy with substitutional elements

$$
\tilde{J}_{k}=-x_{k} \Omega_{k} \nabla \mu_{k}
$$

where

$$
\Omega_{k}=y_{V a} M_{k V a}
$$

$y_{V a}$ is the fraction of vacant lattice sites and $M_{k V a}$ a mobility expressing how often a $k$ atom and a neighboring vacancy exchange places. $x_{k}$ is the mole fraction of $k$ and $\nabla \mu_{k}$ is the gradient in chemical in chemical potential of species $k$. One can then derive the following expression for the diffusivities expressed in the numberfixed frame of reference

$$
D_{k j}=\sum_{i=1}^{n}\left(\delta_{i k}-x_{k}\right) x_{i} \Omega_{i} \frac{\partial \mu_{i}}{\partial x_{j}} V_{m}
$$

where $\delta_{i k}$ is the Kronecker delta, i.e. $\delta_{i k}=1$ if $i=k$ and $\delta_{i k}=0$ otherwise. $V_{m}$ is the molar volume approximated as independent of composition. The numbers $D_{k j}$ form an $n \times n$ matrix. It should be observed that the quantity $\partial \mu_{i} / \partial x_{j}$ is purely thermodynamic and corresponds to the well known thermodynamic factor of Darken. ${ }^{13)}$ This quantity can thus be evaluated from the thermodynamic description of the system. It should be noticed though, that the expressions for the chemical potentials must be given in the form $\mu_{k}\left(x_{1}, x_{2}, \ldots, x_{n}\right)$ in order to get the correct result by means of Eq. (7).

In practical diffusion calculations one eliminates one species from the calculations due to the fact that $\sum_{k=1}^{n} x_{k}=1$ and one rather applies the diffusivity

$$
D_{k j}^{n}=D_{k j}-D_{k n}
$$

where $n$ :th species has been eliminated. The number $D_{k j}^{n}$ form an $(n-1) \times(n-1)$ matrix. From diffusion couple experiments we can only evaluate the $D_{k j}^{n}$ directly and not the $D_{k j}$.

Another sources of information are experiments with radioactive tracers. The tracer diffusivity $D_{k}^{*}$ is directly related to the mobility $\Omega_{k}$ by means of the Einstein relation

$$
D_{k}^{*}=R T \Omega_{k}
$$

Åkermark expressed the $\Omega_{k}$ parameters with the Arrhenius relation

$$
\Omega_{k}=\Omega_{k}^{0} \exp \left(-Q_{k} / R T\right)
$$

and found that the experimental information could be satisfactorily described by assuming linear concentration dependencies of $\Omega_{k}^{0}$ and $Q_{k}$. His results are given in an Appendix.

For alloys containing also interstitial solutes it is more convenient to introduce the concentration variable $u_{k}$ defined as 


$$
u_{k}=\frac{x_{k}}{\sum_{i \in s} x_{i}}
$$

where the symbol $i \in s$ denotes that the summation is performed over the substitutional elements only. Equation (5) is now modified into

$$
\tilde{J}_{k}=-u_{k} \Omega_{k} \nabla \mu_{k}
$$

where $k$ is substitutional and

$$
\tilde{J}_{k}=-u_{k} y_{V a} \Omega_{k} \nabla \mu_{k}
$$

when $k$ is interstitial. $y_{V a}$ is here the fraction of vacant interstitial sites. As a frame of reference we will apply the number-fixed frame of reference with respect to the substitutional elements. I.e.

$$
\sum_{i \in S} J_{i}=0
$$

Equation (7) is now replaced by

$$
D_{k j}=\sum_{i \in s}\left(\delta_{i k}-u_{k}\right) u_{i} \Omega_{i} \frac{\partial \mu_{i}}{\partial u_{j}} V_{S}+\sum_{i \notin s} \delta_{i k} u_{i} y_{V a} \Omega_{i} \frac{\partial \mu_{i}}{\partial u_{j}} V_{S}
$$

where $V_{S}$ is the molar volume of the substitutional atoms. Instead of Eq. (8) we have

$$
\begin{array}{ll}
D_{k j}^{n}=D_{k j}-D_{k n} & \text { when } j \text { is substitutional } \\
D_{k j}^{n}=D_{k j} & \text { when } j \text { is interstitial...... }
\end{array}
$$

\section{Effect of Magnetic Transition in bcc Alloys}

For bcc alloys the occurrence of the transition between para- and ferromagnetic states affects the temperature dependencies of both thermodynamic and kinetic quantities. In the THERMO-CALC system the magnetic effect is represented by an expression initially proposed by Inden ${ }^{14)}$ and later modified by Hillert-Jarl. ${ }^{15)}$ For the Gibbs energy it yields

$$
G_{m}=G_{m}^{p}+\Delta G_{m}^{\mathrm{magn}}
$$

where $G_{m}^{p}$ is the Gibbs energy of the paramagnetic state and $\Delta G_{m}^{\text {magn }}$ is the change in Gibbs energy caused by magnetic ordering and given by

$$
\Delta G_{m}^{\text {magn }}=R T \ln (\beta+1) f(\tau), \quad \tau=T / T_{c}
$$

$\beta$ is the magnetic moment and $T_{c}$ the Curie temperature which are both functions of composition. $f(\tau)$ is given by

$f(\tau)=\frac{1}{A}\left[\frac{79}{140 P}-\frac{474}{497}\left(\frac{1-P}{P}\right)\left(\frac{\tau^{4}}{2}+\frac{\tau^{10}}{15}+\frac{\tau^{16}}{40}\right)\right] \ldots$

for $\tau<1$ and

$$
f(\tau)=\frac{1}{A}\left[\frac{\tau^{-4}}{2}+\frac{\tau^{-14}}{21}+\frac{\tau^{-24}}{60}\right]
$$

for $\tau>1$. $P$ is a structure factor which takes the value 0.28 for fcc structures and 0.40 for bcc structures. The parameter $A$ is given by

$$
A=\frac{518}{1125}+\frac{11692}{15975}\left(\frac{1-P}{P}\right)
$$

The magnetic enthalpy is then given by

$$
\Delta H_{m}^{\mathrm{magn}}=R T_{\mathrm{c}} \ln (\beta+1) f(\tau)
$$

At $T=0 \mathrm{~K}$ we obtain

$$
\Delta H_{m}^{\mathrm{magn}}(0)=R T_{c} \ln (\beta+1) \frac{1}{A} \frac{79}{140 P}
$$

Jönsson ${ }^{16)}$ recently discussed various ways of taking into account the effect of the magnetic ordering upon diffusion. He suggested that one should use a model initially proposed by Braun and Feller-Kniepmeier ${ }^{17}$ because it relates the changes in diffusivities to the magnetic enthalpy, see Eq. (22). Jönsson suggested the following expressions for $\Omega_{k}^{0}$ and $Q_{k}$

$$
\Omega_{k}^{0}=\Omega_{k}^{0 p} \exp (\Lambda \alpha \xi)
$$

and

$$
Q_{k}=Q_{k}^{p}(1+\alpha \xi)
$$

where $\Lambda \approx 6$ and $\alpha \approx 0.3$ in the bcc structure. $\xi$ is a measure of the degree of magnetic order and defined as

$$
\xi=\Delta H_{m}^{\mathrm{magn} /} \Delta H_{m}^{\mathrm{magn}}(0)
$$

$\Omega_{k}^{0 p}$ and $Q_{k}^{p}$ thus denote the frequency factor and the activation energy in the paramagnetic state. When analyzing experimental data on self diffusion of $\mathrm{Fe}$ and tracer diffusion of $\mathrm{Cr}, \mathrm{Ni}, \mathrm{Co}$ in $\mathrm{Fe}$ he found that the model yielded a satisfactory representation of the data. As an example, the tracer diffusion of $\mathrm{Cr}$ in $\mathrm{Fe}$ is shown in Fig. 1.

For interstitial elements the situation is somewhat obscure. For carbon a strong effect is observed although the above formalism does not fit the experimental data. Instead $\AA$ gren $^{2)}$ applied a formalism in which the same activation energy is applied in the paramagnetic and ferromagnetic states. The expression given in Ref. 2) contains a printing error and below the correct expression is given

$$
\begin{aligned}
& R T \Omega_{C}^{\alpha}=2 \cdot 10^{-6} \cdot e^{-10115 / T} \\
& \cdot \exp \left\{0.5898\left[1+\frac{2}{\pi} \arctan \left(14.985-\frac{15309}{T}\right)\right]\right\} \\
& \left(\mathrm{m}^{2} \mathrm{~s}^{-1}\right) \ldots \ldots \ldots
\end{aligned}
$$

For nitrogen there seem to be no observable effect at all.

\section{The DICTRA Package}

The basic structure of the DICTRA software is shown in Fig. 2. There are 3 main modules, one for solving the diffusion equations, i.e. Eq. (4), inside each phase, and one for calculating the local equilibrium at the phase interface. The third module sets up and solves the flux-balance equations, i.e. Eq. (1), in order to obtain the transformation rates. The position of the phase interface is subsequently obtained by integration. In addition the package contains a module for post processing of the output which enables the user to obtain various listings and plottings. Some more details concerning the software are given in Ref. 18).

The software has now been implemented in VMS 


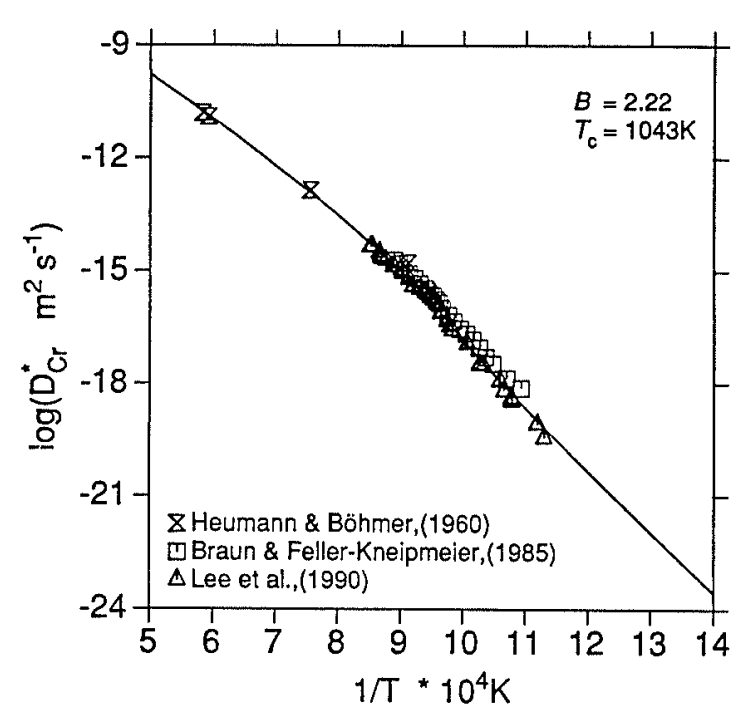

Fig. 1. Tracer diffusion of chromium in bcc iron. The symbols denote experimental data (from Ref. 16)).

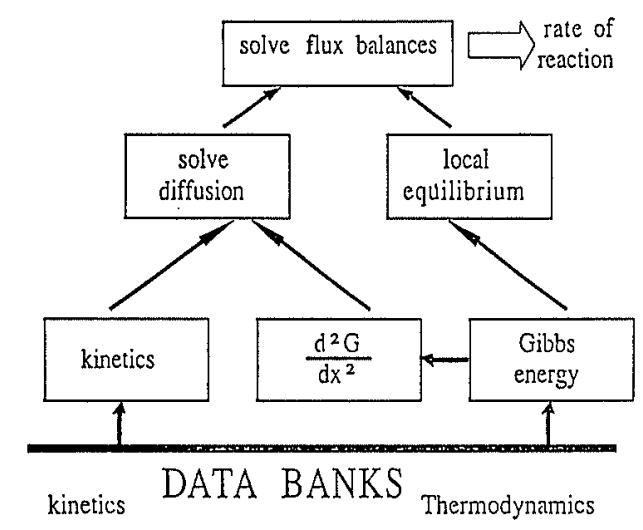

Fig. 2. Basic structure of the DICTRA program.

environment on the VAX system and in UNIX on the SUN work stations.

\section{Example of Simulations}

\subsection{The Austenite-Ferrite Transformation during Cool- ing}

There is no extra difficulty to perform the simulations under varying temperatures. Jönsson ${ }^{19)}$ recently performed a series of simulations of the growth of grainboundary ferrite in low-alloy steels. Figure 3 taken from Ref. 19 shows how the fraction of ferrite varies in an $\mathrm{Fe}-0.2 \mathrm{wt} \% \mathrm{C}$ alloy with various additions. The cooling rate is $0.1 \mathrm{~K} \mathrm{~s}^{-1}$. By performing a series of calculations for a given alloy but for different cooling rates one can construct a CCT diagram. As an example, a CCT diagram calculated by Jönsson for the $\mathrm{Fe}-0.2 \mathrm{wt} \% \mathrm{C}$ alloy is shown in Fig. 4. The calculations were performed for a grainsize of $50 \mu \mathrm{m}$ and assuming nucleation of ferrite as soon as it becomes thermodynamically stable. The time is taken as zero when $A_{3}=1110 \mathrm{~K}\left(837^{\circ} \mathrm{C}\right)$ is passed.

\subsection{Dissolution of Cementite in Low-alloy Steels}

The dissolution of carbides play an important role during austenitizing in hardening of steels. Liu et al. ${ }^{20)}$ recently made an experimental investigation of cementite

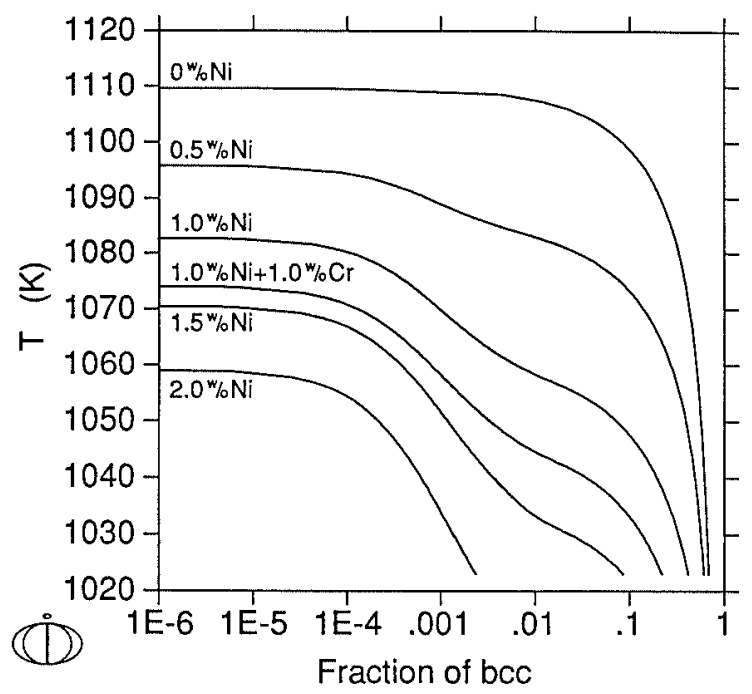

Fig. 3. Simulation of ferrite growth during continuous cooling. Temperature $v s$. fraction of ferrite formed for $\mathrm{Fe}-0.2 \mathrm{wt} \% \mathrm{C}$ alloys cooled at $0.1 \mathrm{~K} \mathrm{~s}^{-1}$. Labels indicate amount of alloying elements in addition to Fe and C. From Ref. 19).

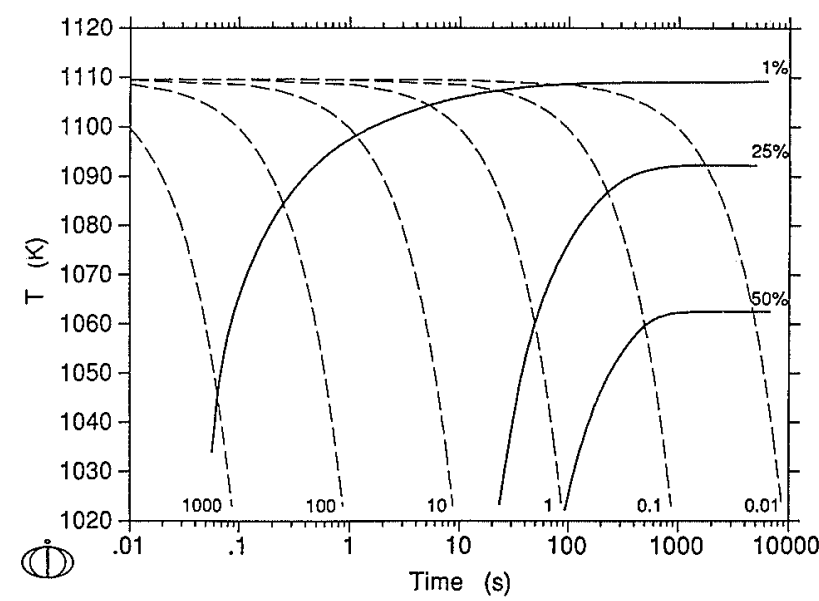

Fig. 4. Simulation of ferrite growth during continuous cooling. Calculated CCT diagram for grain-boundary ferrite in $\mathrm{Fe}-0.2 \mathrm{wt} \% \mathrm{C}$ alloy. From Ref. 19).

dissolution during austenitizing of an $\mathrm{Fe}-2 \mathrm{wt} \% \mathrm{Cr}-$ $0.85 \mathrm{wt} \% \mathrm{C}$ alloy. Their experimental results were compared with calculations using the DICTRA program assuming that the cementite particles are spherical. As an example the calculated and experimental volume fractions of cementite are shown in Fig. 5 taken from Ref. 20.

\subsection{Dissolution of $M_{23} C_{6}$ in Martensitic Stainless Steels}

The austenitizing of more high-alloy steels, like martensitic stainless steels containing 13-14 wt \% Cr and $0.6-0.7 \mathrm{wt} \% \mathrm{C}$ has been studied by Omsén and Liljestrand ${ }^{21)}$ and by Gullberg. ${ }^{22)}$ The major difference between the high-alloy and the low-alloy steels is that in the former more complex carbides form and since they hold a high $\mathrm{Cr}$-content longer heat treatment times may be required in order to achieve a homogeneous austenite. Höglund ${ }^{23)}$ recently applied the DICTRA program in order to simulate the dissolution of $\mathrm{M}_{23} \mathrm{C}_{6}$ particles 


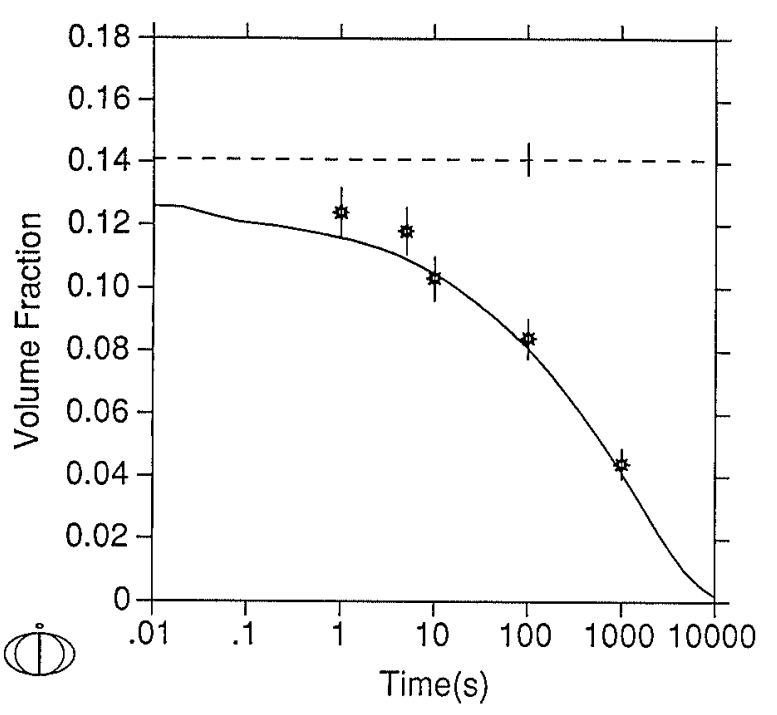

Fig. 5. Calculated volume fraction of cementite $v s$. austenitizing time at $910^{\circ} \mathrm{C}$ For $2 \mathrm{wt} \% \mathrm{Cr}-0.85 \mathrm{wt} \% \mathrm{C}$ steel. Symbols are experiments. From Ref. 20).

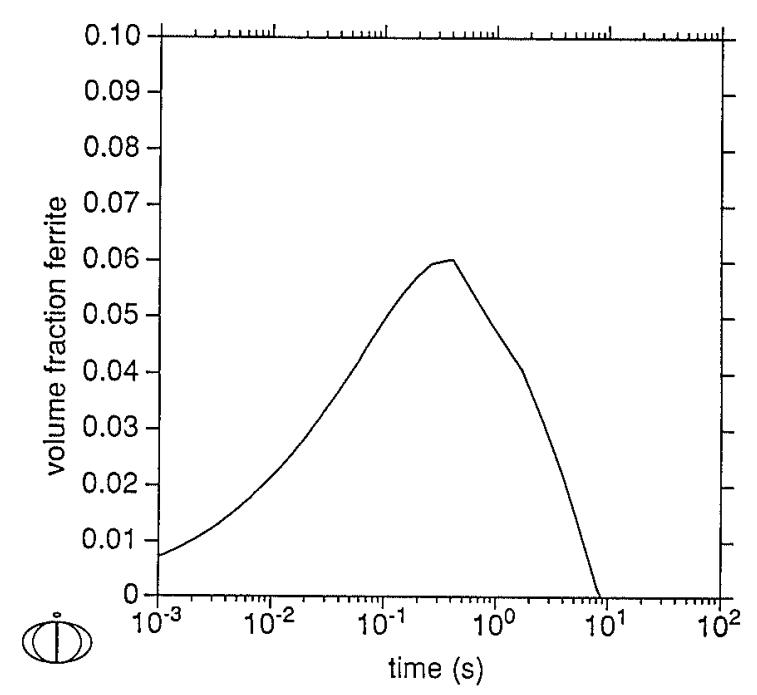

Fig. 6. Ferrite formation upon dissolution of $\mathrm{M}_{23} \mathrm{C}_{6}$ during austenitizing at $1000^{\circ} \mathrm{C}$ of $13 \mathrm{wt} \% \mathrm{Cr}-0.7 \mathrm{wt} \% \mathrm{C}$ martensitic stainless steel. From Ref. 23).

during austenitizing of martensitic stainless steels. The thermodynamic description was taken from Hillert and $\mathrm{Qiu}^{3)}$ and the diffusivities from $\AA$ kermark ${ }^{11)}$ and from Àgren. ${ }^{24)}$ In one simulation a steel with $13 \mathrm{wt} \% \mathrm{Cr}$ and $0.7 \mathrm{wt} \% \mathrm{C}$ was austenitized at $1000^{\circ} \mathrm{C}$. The particles were assumed spherical with an initial diameter of $0.25 \mu \mathrm{m}$ and a $\mathrm{Cr}$ content corresponding to equilibrium partitioning at an annealing temperature of $800^{\circ} \mathrm{C}$.

The calculations show that a thin layer of ferrite will form in the very early stages of the reaction and then disappear. This result is in agreement with the TEMobservations by Omsén and Liljestrand. Figure 6 shows the calculated fraction of ferrite as a function of time. However, a simulation performed under the constraint that the ferrite may not form shows that the ferrite formation does not really have any effect on the alloy content of the austenite at times longer than $1 \mathrm{~min}$.

Figure 7 shows a comparison with experimental data from Gullberg for a steel with $14 \mathrm{wt} \% \mathrm{Cr}$ and $0.6 \mathrm{wt} \%$

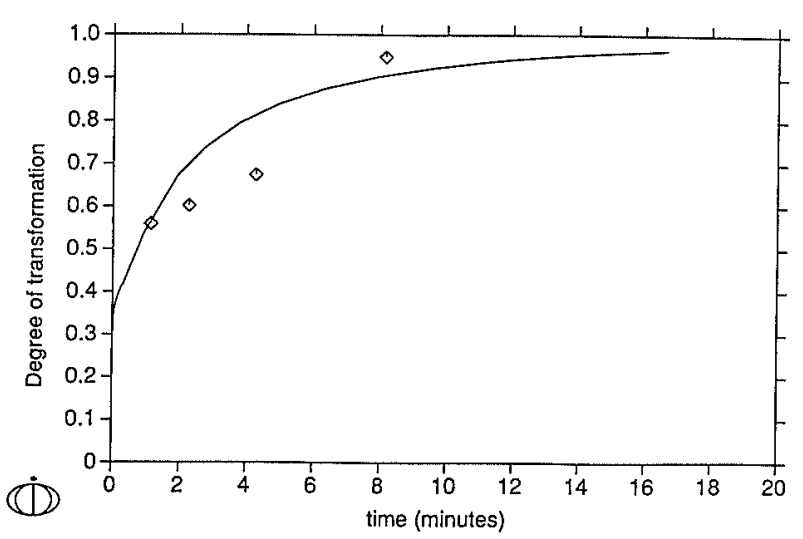

Fig. 7. Degree of $\mathrm{M}_{23} \mathrm{C}_{6}$ dissolution during austentizing at I $100^{\circ} \mathrm{C}$ of $14 \mathrm{wt} \% \mathrm{Cr}-0.6 \mathrm{wt} \% \mathrm{C}$ martensitic stainless steel. Solid line: simulations from Ref. 23). Symbols: experimental data from Ref. 22).

C austenitized at $1100^{\circ} \mathrm{C}$. The degree of transformation is defined as

$$
\frac{\text { change in volume fraction }}{\text { change in volume fraction if equilibrated }}
$$

The agreement is not perfect but it is encouraging and it should be kept in mind that the calculation contains no adjustable parameters.

\section{Prediction of Shape}

The problem of predicting the actual shape of a growing particle on a physical basis is much more difficult than calculating the growth rate for a given shape. In general a 3-dimensional diffusion problem must be solved. Such approaches are now being applied when solving the heat-flow equation. Mathematically this would correspond to isothermal diffusion in a binary alloy. The multicomponent diffusion problem has not yet been treated in a general 3-dimensional geometry. In addition surface tension and stresses caused by coherency or volume changes play important roles in controlling the shape of growing particles. The kinetics of the interface reactions themselves may also be important under certain conditions. It is certainly a challenge to apply the multicomponent thermodynamics and kinetics to more complex geometries.

\section{Conclusive Remarks}

The recent development in computer simulations of multicomponent diffusional reactions has been discussed. The importance of assessing and compiling multicomponent diffusion data has been addressed. It is suggested that the experimental data on multicomponent diffusion should be analyzed in a way similar to thermodynamic data by means of the CALPHAD technique. The results of some recent simulations are shown. These results are quite encouraging and one may conclude that it is now possible to predict transformation rates in complex alloys from basic thermodynamic and kinetic information provided that the morphology is simple enough. The prediction of shapes is much more complex and should now be a challenge for 
the development during the 90:s. However, it must be emphasized that much work is now needed on experimental determination and assessment of multicomponent diffusion data.

\section{Acknowledgments}

The author expresses his thanks to Dr. Björn Jönsson and $\mathrm{Mr}$. Lars Höglund for stimulating discussions. Long-term support from the Swedish Board for Technical Development and from the Volkswagen Stiftung is gratefully acknowledged.

\section{REFERENCES}

1) J. Agren: J. Phys. Chem. Solids, 43 (1982), 385.

2) J. Ågren: Acta Metall., 30 (1982), 841 .

3) M. Hillert and C. Qiu: Metall. Trans. A, 21A (1990), 1673.

4) K. Frisk: Metall. Trans. A, 21A (1990), 2477.

5) W. Huang: Z. Metallkde., Bd 82 (1991), H.5, 391.

6) P. Gustafson: Z. Metallkde., Bd 79 (1988), H.7, 42

7) B. Sundman, B. Jansson and J-O Andersson: CALPHAD, 9 (1985), 153.

8) J. Ågren: Scand. J. Metall., 20 (1991) 86-92.

9) J. Ågren: Internal Report, series D 84, 1987, Div. of Phys. Metall., Royal Inst. Tech., S-100 44 Stockholm, Sweden.

10) J-O. Andersson and J. Ågren: TRITA-MAC 0436, May 1990 Internal Report, Div. of Phys. Metall., Royal Inst. Tech., S-100 44 Stockholm, Sweden.

11) T. Åkermark: Diploma Work 1991, Div. of Phys. Metall., Royal Inst. Tech., S-100 44 Stockholm, Sweden.

12) J. S. Kirkaldy and D. J. Young: Diffusion in the Condensed State, Inst. of Met., London, (1987)

13) L. S. Darken: Trans. AIME, 175 (1948), 184.

14) G. Inden: Project Meeting CALPHAD V, Max Planck Institut für Eisenforschung, Düsseldorf, 21-25 June 1976.

15) M. Hillert and M. Jarl: CALPHAD, 2 (1978), 227.

16) B. Jönsson: TRITA-MAC 0451, Jan. 1991, Internal Report, Div. of Phys. Metall., Royal Inst. Tech., S-100 44 Stockholm, Sweden.
17) R. Braun and M. Feller-Kniepmeier: Scr. Metall., 20 (1986), 7.

18) J.-O. Andersson, L. Höglund, B. Jönsson and J. Ågren: Fundamentals and Applications of Ternary Diffusion, ed. by $G$. R. Purdy, Pergamon Press, New York, (1990), 153.

19) B. Jönsson: Internal Report, series D 101, 1991, Div. of Phys Metall. Royal Inst. Tech., S-100 44 Stockholm, Sweden.

20) Z.-K. Liu, L. Höglund, B. Jönsson and J. Ågren: Metall. Trans. $A, 22 \mathrm{~A}(1991), 1745$.

21) A. Omsén and L.-G. Liljestrand: Scand.J. Metall., 1(1972), 241.

22) R. Gullberg: J. Iron Steel Inst., 211 (1973), 59.

23) L. Höglund: to be published.

24) J. Ågren: Scr. Metall., 20 (1986), 1507.

\section{Appendix}

For diffusion of iron in fcc $\mathrm{Fe}-\mathrm{Cr}-\mathrm{Ni}$ alloys the following expressions are obtained:

$$
\begin{gathered}
\frac{Q_{\mathrm{Fe}}}{R}=35880 x_{\mathrm{Fe}}+34410 x_{\mathrm{Ni}}+36130 x_{\mathrm{Cr}} \\
R T \Omega_{\mathrm{Fe}}^{0}=3.202 \cdot 10^{-4} x_{\mathrm{Fe}}+3.164 \cdot 10^{-4} x_{\mathrm{Ni}} \\
+2.364 \cdot 10^{-4} x_{\mathrm{Cr}}\left(\mathrm{m}^{2} \mathrm{~s}^{-1}\right)
\end{gathered}
$$

For diffusion of nickel in $\mathrm{Fe}-\mathrm{Cr}-\mathrm{Ni}$ alloys:

$$
\begin{gathered}
\frac{Q_{\mathrm{Ni}}}{R}=35650 x_{\mathrm{Fe}}+34600 x_{\mathrm{Ni}}+38010 x_{\mathrm{Cr}} \\
R T \Omega_{\mathrm{Ni}}^{0}=1.111 \cdot 10^{-4} x_{\mathrm{Fe}}+3.808 \cdot 10^{-4} x_{\mathrm{Ni}} \\
+1.542 \cdot 10^{-4} x_{\mathrm{Cr}}\left(\mathrm{m}^{2} \mathrm{~s}^{-1}\right)
\end{gathered}
$$

For diffusion of chromium in $\mathrm{Fe}-\mathrm{Cr}-\mathrm{Ni}$ alloys:

$$
\begin{gathered}
\frac{Q_{\mathrm{Cr}}}{R}=36100 x_{\mathrm{Fe}}+32080 x_{\mathrm{Ni}}+34620 x_{\mathrm{Cr}} \\
R T \Omega_{\mathrm{Cr}}^{0}= \\
\quad 2.747 \cdot 10^{-4} x_{\mathrm{Fe}}+1.117 \cdot 10^{-4} x_{\mathrm{Ni}} \\
+1.444 \cdot 10^{-4} x_{\mathrm{Cr}}\left(\mathrm{m}^{2} \mathrm{~s}^{-1}\right)
\end{gathered}
$$

